\title{
Pretransplant Physical Activity Predicts All-Cause Mortality in Kidney Transplant Recipients
}

\author{
Sylvia E. Rosas ${ }^{a, b}$ Peter P. Reese $^{b, c}$ Yonghong Huan $^{d}$ Cataldo Doria $^{d}$ \\ Philip T. Cochetti ${ }^{a} \quad$ Alden Doyle $^{e}$ \\ a Philadelphia Veterans Administration Medical Center, ${ }^{b}$ Department of Medicine and ${ }^{\mathrm{C} C e n t e r}$ for Clinical \\ Epidemiology and Biostatistics, Department of Biostatistics and Epidemiology, University of Pennsylvania, \\ ${ }^{\mathrm{d}}$ Thomas Jefferson University, and ${ }^{\text {e}}$ Drexel University, Philadelphia, Pa., USA
}

\section{Key Words}

Kidney transplantation • Mortality • Physical activity

\begin{abstract}
Background: Low physical activity (PA) has been associated with higher rates of cardiovascular disease (CVD) and mortality in the general population. Despite the benefits of kidney transplantation, kidney transplant recipients (KTRs) remain at elevated risk for CVD and mortality compared to individuals without kidney disease. Methods: A prospective cohort of 507 adult KTRs from three academic centers completed the Physical Activity Scale for the Elderly (PASE) at transplantation. PASE scores were divided into tertiles. Results: PA was lower with older age, history of CVD, smoking, and diabetes. During the median 8-year follow-up period, 128 individuals died, among whom 101 had a functioning allograft. In multivariable Cox regression for all-cause mortality, greater PA was strongly associated with better survival (HR: 0.52 for most active vs. inactive tertiles, 95\% Cl: 0.31-0.87, $\mathrm{p}=0.01$ ). Secondary analyses, in which (1) death with a functioning graft was the primary outcome, and (2) PASE scores were converted to the metabolic equivalent of task, revealed similar results. We did not find an association between change of PA after transplantation and mortality. Conclusions: PA at the time of kidney transplantation is a strong predictor of all-
\end{abstract}

\section{KARGER}

(C) 2011 S. Karger AG, Basel

Fax +41613061234 E-Mail karger@karger.ch www.karger.com www.karger.com/ajn cause mortality and death with graft function. Evaluation of PA level among kidney transplant candidates may be a useful method to risk-stratify patients for survival after kidney transplantation. Kidney transplant candidates and recipients should also be encouraged to be physically active.

Copyright $\odot 2011$ S. Karger AG, Basel

\section{Introduction}

Compared to chronic dialysis, kidney transplantation substantially decreases the risk of death and cardiovascular disease (CVD) events, and improves quality of life for most individuals with end-stage kidney disease (ESKD). Given the scarcity of kidney allografts, there is increasing interest in identifying patient characteristics before transplantation that predict posttransplant survival, as well as matching projected survival of kidney transplant candidates to projected allograft survival. Studies of diverse populations suggest that physical activity (PA) independently predicts mortality. Greater PA may directly improve overall health and cardiovascular fitness through improvements in blood pressure, lipids, insulin sensitivity, and systemic inflammation.

Kidney transplant recipients (KTRs) have fourfold increases in pooled nonfatal and fatal CVD incidence when 
compared to the general population [1]. This markedly elevated risk of CVD has been attributed to diverse pathological changes associated with advancing kidney disease, including hypertension, left ventricular hypertrophy, anemia, coronary calcification, and endothelial dysfunction [2].

Most studies of PA in the ESKD population have involved dialysis populations, while a few have focused on PA levels among KTRs. In a small study, 32 KTRs selfreported their PA at 1, 3, 6, 12, and 60 months [3]. KTRs had PA similar to elderly individuals in the general population, and PA levels increased approximately $30 \%$ of their baseline by 1 year after transplantation.

The aims of this study were to (1) assess pretransplant PA in a prospective cohort of incident and racially diverse KTRs, (2) identify predictors of pretransplant PA, (3) evaluate changes in PA after transplantation, and (4) determine the association between pretransplant PA with all-cause mortality and death with graft function.

\section{Materials and Methods}

We conducted a prospective cohort study of KTRs, who were recruited during their initial transplant hospitalization or shortly following their transplant. Recipients of multiorgan transplants were excluded, except for kidney-pancreas recipients. The primary outcome was mortality and the primary exposure was pretransplant PA.

Institutional review board approval was obtained. Informed written consent was collected from all participants.

\section{Physical Activity}

Pretransplant PA was measured using the Physical Activity Scale for the Elderly (PASE) questionnaire in recipients recruited between August 2000 and June 2004. The PASE is a 10-item instrument which assesses PA in the domains of leisure, household, and work activities, and takes $5 \mathrm{~min}$ to complete. Respondents were instructed to report their PA level for the prior week. Though originally designed for individuals over 65 years of age and developed with reference to accelerometer data [4], the PASE has been validated in cohorts of nonelderly, chronically ill, and sedentary individuals, as well as patients with ESKD $[4,5]$. The PASE score has also been correlated with physiological measurements such as $\mathrm{VO}_{2}$ [6]. The PASE score is computed by multiplying the duration of time spent in each activity in hours per day over a 7-day period by the respective weights and summing the scores of all activities. Because PASE scores were not normally distributed, we empirically divided recipients into tertiles of PASE scores. We also evaluated PASE as a continuous variable and as the natural log of the PASE score.

In additional secondary analysis using established methods, we calculated metabolic equivalents of task using the responses from the PASE questionnaire [7]. A subset of recipients had a second questionnaire completed a year after transplantation.
Delayed Graft Function and Mortality

Delayed graft function was defined as the need for dialysis during the first week after transplantation. Mortality was assessed through medical records and through the Social Security Death Index. Survival time was defined as time from transplant to death in years or March 31, 2011. Death with graft function survival time was defined as time from transplant to death in years or March 31, 2011 with censoring on date of allograft failure.

\section{Statistical Analysis}

All data analyses were performed using Stata Version 10.1 (Stata Corp., College Station, Tex., USA). Since PASE scores were not normally distributed, we created tertiles of PA, stratified by gender as per other reports [8]. We determined associations between PASE tertile and other participant attributes using ANOVA for continuous variables or $\chi^{2}$ for categorical variables. In addition, we report total PASE scores categorized as limited $(<50.0)$, low (50.1-200.0), and high (>200.0) PA to allow comparisons with previously reported PASE results based on cross-sectional studies [9]. We evaluated the change of PA after transplantation using previously described methods [10].

Kaplan-Meier survival analysis was used to evaluate mortality and death with graft function based on PASE score tertile. Significance was evaluated using the log-rank test.

Multivariable Cox proportional hazard models were used to evaluate risk factors associated with mortality and death with graft function. Both unadjusted and adjusted models were used to identify factors that were predictive of mortality events in multivariate models with PASE score. Appropriate diagnostics were performed to ensure that the proportionality assumption was not violated in the implementation of the Cox model. We selected a priori variables that had been previously associated with survival as well as considered covariates with $\mathrm{p}<0.20$ in univariate models for inclusion in the survival analyses. Covariates that were considered included recipient and donor age, race, gender, education, income, transplant type (deceased vs. living donor), HDL, triglycerides, Apo A1, BMI, history of smoking, CVD, diabetes, and ESKD vintage. Income was not entered into the final multivariable models due to underreporting.

A small proportion of patients had missing data (smoking history: $\mathrm{n}=25$, dialysis vintage: $\mathrm{n}=17$, BMI: $\mathrm{n}=14$, delayed graft function: $\mathrm{n}=2$ ). In order to explore the potential for bias due to nondifferential missing data, we performed secondary analyses using the multiple imputation by chained equations (MICE) procedure, and using sensitivity analyses. A total of 10 imputed datasets were created. Results were similar to those presented with all the nonmissing data in table 1.

\section{Results}

Five hundred and fifteen recipients completed the PASE questionnaire within 2 weeks of transplantation. The median time from transplant to questionnaire completion was 3 days. Due to missing data, eight PASE scores could not be calculated. Therefore, the cohort was comprised of 507 recipients with complete questionnaires. 
Table 1. Recipient characteristics by PA gender-stratified tertiles

\begin{tabular}{|c|c|c|c|c|c|c|c|}
\hline & $\mathrm{n}^{1}$ & Inactive & $\mathrm{n}^{1}$ & Moderate & $\mathrm{n}^{1}$ & Active & $\mathrm{p}$ \\
\hline Mean age, years & 169 & $51.4 \pm 13.1$ & 166 & $47.3 \pm 12.7$ & 172 & $44.8 \pm 11.6$ & $<0.001$ \\
\hline Mean BMI & 157 & $27.9 \pm 5.8$ & 158 & $27.3 \pm 5.7$ & 154 & $26.8 \pm 6.5$ & 0.33 \\
\hline African American & 169 & $58(34.3)$ & 166 & $58(34.9)$ & 172 & $45(26.2)$ & 0.15 \\
\hline Male & 169 & $103(61.0)$ & 166 & $102(61.4)$ & 172 & $104(60.5)$ & 0.98 \\
\hline College-educated & 156 & $60(38.5)$ & 154 & $47(30.5)$ & 167 & $74(44.3)$ & 0.04 \\
\hline Married & 156 & $98(62.8)$ & 156 & $102(65.4)$ & 167 & $103(61.7)$ & 0.78 \\
\hline Income USD $>40,000$ & 121 & $59(48.8)$ & 115 & $55(47.8)$ & 129 & $63(48.8)$ & 0.99 \\
\hline Living donor transplant & 169 & $55(32.5)$ & 166 & $64(38.6)$ & 172 & $68(39.5)$ & 0.35 \\
\hline CVD & 160 & $52(32.7)$ & 157 & $46(29.3)$ & 168 & $42(25)$ & 0.31 \\
\hline Diabetes & 169 & $71(42)$ & 166 & $48(28.9)$ & 172 & $40(23.3)$ & 0.001 \\
\hline Hypertension & 165 & $157(95.2)$ & 162 & $158(97.5)$ & 172 & $161(93.6)$ & 0.23 \\
\hline Ever smoked & 158 & $85(53.8)$ & 157 & $83(52.9)$ & 167 & $69(41.3)$ & 0.04 \\
\hline Prior dialysis & 162 & $137(84.6)$ & 162 & $132(81.5)$ & 166 & $137(82.5)$ & 0.76 \\
\hline Graft failure & 169 & $23(13.6)$ & 166 & $26(15.7)$ & 172 & $17(9.9)$ & 0.28 \\
\hline $\mathrm{HDL} \geq 40, \mathrm{mg} / \mathrm{dl}$ & 91 & $38(41.8)$ & 98 & $47(48)$ & 102 & $53(52)$ & 0.36 \\
\hline $\mathrm{HDL}, \mathrm{mg} / \mathrm{dl}$ & 91 & $43.5 \pm 20.3$ & 98 & $41.6 \pm 13.9$ & 102 & $42.5 \pm 13.0$ & 0.72 \\
\hline $\mathrm{LDL}, \mathrm{mg} / \mathrm{dl}$ & 86 & $93.1 \pm 38.1$ & 92 & $92.0 \pm 34.1$ & 100 & $99.4 \pm 32.8$ & 0.29 \\
\hline Total cholesterol, mg/dl & 91 & $169.0 \pm 50.2$ & 98 & $169.1 \pm 41.4$ & 102 & $172.9 \pm 41.2$ & 0.77 \\
\hline Triglycerides, mg/dl & 91 & $167.5 \pm 118.7$ & 98 & $178.9 \pm 131.8$ & 102 & $153.8 \pm 84.7$ & 0.29 \\
\hline Creatinine at transplant, $\mathrm{mg} / \mathrm{dl}$ & 158 & $7.97 \pm 3.41$ & 153 & $8.01 \pm 3.02$ & 157 & $8.70 \pm 3.39$ & 0.09 \\
\hline
\end{tabular}

Values in parentheses represent percentages.

1 Total numbers may vary due to missing data.

Table 1 describes the characteristics of recipients according to PASE score tertile. The majority of the cohort was male (61.0\%), non-African American (68.2\%), and received deceased donor transplants (63.1\%). The mean $( \pm S D)$ age was $47.8 \pm 12.8$ years. Kidney disease was most commonly attributed to hypertension (67.3\%), diabetes $(27.1 \%)$, and polycystic kidney disease (14.3\%). Over one third of the participants had a history of cardiovascular events pretransplant and $31.4 \%$ had diabetes.

\section{PA Score}

Figure 1 displays the distribution of the PASE scores. The median PASE score was 92.7, with a range of 0-415.96 (25-75th interquartile range: 58.6-141) and a mean of $105.3 \pm 70.2$. The average PASE score was $103.3 \pm 71.5$ for men and $108.3 \pm 68.1$ for women. Recipients with higher scores were younger, less likely to have diabetes, and less likely to smoke. Using the activity category, $20 \%$ of recipients were in the limited category while the majority was in the low category (70.2\%). Only $9.6 \%$ were in the high activity category.

With regard to leisure activity, $14.6 \%$ reported participating in light sports and $18.7 \%$ reported endurance

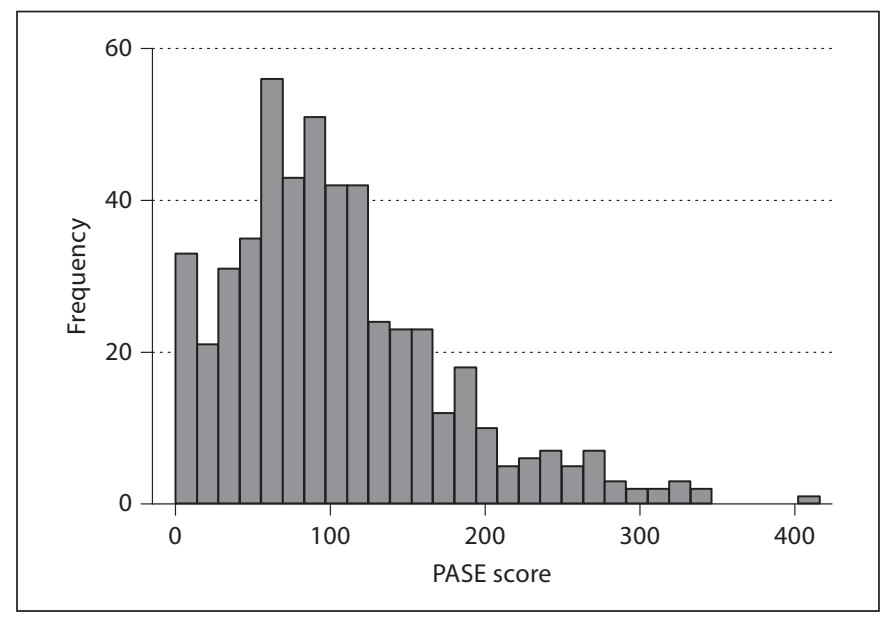

Fig. 1. Distribution of PASE scores in the kidney transplant cohort.

training at least once per week. About a third of the recipients reported volunteering or working outside of the home at least part-time. There were no significant gender differences in the work (17.3 vs. 19.0, $\mathrm{p}=0.59$ ), leisure (24.3 vs. $21.5, \mathrm{p}=0.36$ ), and household subscores (61.8 

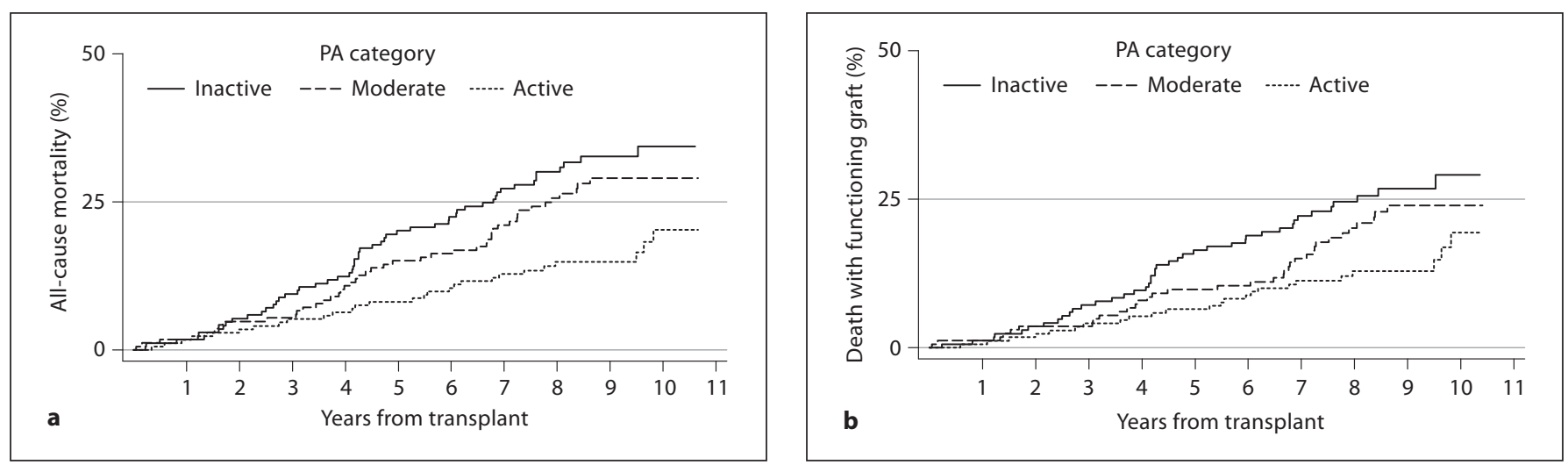

Fig. 2. a Kaplan-Meier curves of all-cause mortality according to gender-stratified tertiles of PA $(p=0.002)$. b Kaplan-Meier curves of death with a functioning graft according to gender-stratified tertiles of PA $(p=0.02)$.

vs. 67.8, $\mathrm{p}=0.12$ ) between men and women (online suppl. table 1; for all online suppl. material, see www. karger.com/doi/10.1159/000334732).

\section{$P A$ and Mortality}

Median follow-up from transplantation was 8.4 years (interquartile range: 7.2-9.6), with a total of 3,990 personyears of observation. There were 128 deaths during the study period; among these, 101 of the deceased had functioning grafts. PASE scores were significantly higher among recipients who survived versus those who died $(111.7 \pm 71.6$ vs. $86.1 \pm 62.0, \mathrm{p}<0.001)$. Longer survival was also associated with younger age $(46.0 \pm 12.7$ vs. 53.1 \pm 11.0 years, $\mathrm{p}<0.001)$ and shorter ESKD vintage $(2.64$ \pm 3.3 vs. $4.5 \pm 5.5$ years, $p<0.001)$. In addition, recipients who survived were less likely to have diabetes (26.4 vs. $46.1 \%, \mathrm{p}<0.001)$, CVD (24.9 vs. $42 \%, \mathrm{p}<0.001)$, and a smoking history (44.5 vs. $63.8 \%, \mathrm{p}<0.001)$, and were more likely to have an income USD $>40,000 /$ year (82.5 vs. $67.6 \%, p=0.001)$. Recipients who survived were less likely to have deceased donor transplants $(60.2$ vs. $71.9 \%$, p = 0.02). There were no differences in BMI, race, and sex between the recipients who survived and those who expired.

There was also a graded association between activity category and death. In the limited category $36.3 \%$ of recipients died, while in the low category it was $23.3 \%$ and in the high category it was $16.3 \%(\mathrm{p}=0.01)$.

Table 2 depicts the multivariable models. Model 1 was adjusted for demographic variables and Model 2 was additionally adjusted for comorbidities. The highest PASE tertile (active group) had significantly longer survival after multivariable adjustment for demographic and clinical attributes as well as comorbidities. In our analysis by
PASE score tertile, increasing age [lower tertile $=$ reference; middle tertile $=$ HR: $2.6(1.4-4.8), \mathrm{p}=0.002$; higher tertile $=$ HR: 3.5 (1.9-6.2), $\mathrm{p}<0.001$ ], diabetes [HR: 1.7 (1.1-2.5), $\mathrm{p}=0.01$ ], and ESKD vintage [1st quartile (lower) $=$ reference; 2 nd quartile $=$ HR: $1.5(0.82-2.9), p=0.18$; 3rd quartile = HR: 2.1 (1.2-3.8), $\mathrm{p}=0.02$; 4 th quartile (high) = HR: 3.3 (1.8-6.1), p < 0.001] were also significantly associated with all-cause mortality. Delayed graft function was marginally associated with mortality [HR: $1.68(0.99-2.8), \mathrm{p}=0.05]$.

Sensitivity analyses revealed similar results when the primary exposure was the PASE score as a continuous measure or as the natural log of the PASE score, and when the primary exposure was metabolic equivalent of task.

Figure 2a represents the Kaplan-Meier estimates of cumulative overall mortality for kidney recipients by gender-stratified PASE score tertile $(\mathrm{p}<0.01)$. Figure $2 \mathrm{~b}$ represents the Kaplan-Meier estimates of cumulative overall death with graft function stratified by PASE score tertile $(\mathrm{p}=0.02)$.

In a subset of participants $(\mathrm{n}=291)$ with HDL measurements on the date of transplant, an HDL level $\geq 40$ $\mathrm{mg} / \mathrm{dl}$ was also predictive of improved survival [HR: 0.45 (0.25-0.82), $\mathrm{p}=0.01]$. The relationship between PA and mortality was unchanged.

\section{Change in $P A$}

Follow-up measurements were available in 290 recipients a median of 11.1 months after the first questionnaires. The recipients with a follow-up questionnaire data had similar baseline PASE scores compared to those without it (median: 91 vs. 93.6, $\mathrm{p}=0.82$ ). While most characteristics were similar to the complete cohort, those with a follow-up 
Table 2. Association of PA with all-cause mortality and death with functioning graft

\begin{tabular}{|c|c|c|c|c|c|c|c|c|c|c|c|c|}
\hline \multirow[t]{2}{*}{ PA measure } & \multicolumn{4}{|c|}{ Unadjusted $(\mathrm{n}=507)$} & \multicolumn{4}{|c|}{ Model $1^{1}(\mathrm{n}=507)$} & \multicolumn{4}{|c|}{ Model $2^{2}(\mathrm{n}=454)$} \\
\hline & HR & \multicolumn{2}{|c|}{$95 \% \mathrm{CI}$} & $\mathrm{p}$ & HR & \multicolumn{2}{|c|}{$95 \% \mathrm{CI}$} & $\mathrm{p}$ & HR & \multicolumn{2}{|c|}{$95 \% \mathrm{CI}$} & $\mathrm{p}$ \\
\hline \multicolumn{13}{|c|}{ All-cause mortality $(n=128)$} \\
\hline METS $^{3}$ & 0.91 & \multicolumn{2}{|c|}{$0.87-0.96$} & $<0.001$ & 0.93 & \multicolumn{2}{|c|}{$0.88-0.97$} & 0.002 & 0.94 & \multicolumn{2}{|c|}{$0.88-0.99$} & 0.02 \\
\hline PASE score ${ }^{3}$ & 0.95 & \multicolumn{2}{|c|}{$0.92-0.97$} & $<0.001$ & 0.96 & \multicolumn{2}{|c|}{$0.93-0.99$} & 0.005 & 0.96 & \multicolumn{2}{|c|}{$0.92-0.99$} & 0.005 \\
\hline $\log$ PASE & 0.89 & \multicolumn{2}{|c|}{$0.80-0.99$} & 0.04 & 0.89 & \multicolumn{2}{|c|}{$0.80-0.99$} & 0.04 & 0.88 & \multicolumn{2}{|c|}{$0.78-0.99$} & 0.04 \\
\hline \multicolumn{13}{|l|}{ PASE tertile } \\
\hline Inactive & ref. & \multirow{2}{*}{\multicolumn{2}{|c|}{$0.55-1.20$}} & & ref. & & & & ref. & & & \\
\hline Moderate & 0.81 & & & 0.31 & 0.91 & \multicolumn{2}{|c|}{$0.61-1.36$} & 0.65 & 0.87 & \multicolumn{2}{|c|}{$0.56-1.35$} & 0.53 \\
\hline Active & 0.45 & \multicolumn{2}{|c|}{$0.29-0.72$} & 0.001 & 0.53 & \multicolumn{2}{|c|}{$0.33-0.84$} & 0.01 & 0.52 & \multicolumn{2}{|c|}{$0.31-0.87$} & 0.01 \\
\hline \multicolumn{13}{|c|}{ Death with functioning graft $(n=101)$} \\
\hline METS $^{3}$ & 0.92 & 0.87 & 0.97 & 0.003 & 0.93 & 0.88 & 0.98 & 0.01 & 0.94 & 0.89 & 1.01 & 0.07 \\
\hline PASE score ${ }^{3}$ & 0.95 & 0.92 & 0.98 & 0.002 & 0.96 & 0.93 & 0.99 & 0.01 & 0.95 & 0.92 & 0.99 & 0.01 \\
\hline $\log$ PASE & 0.90 & 0.79 & 1.02 & 0.09 & 0.89 & 0.78 & 1.02 & 0.08 & 0.87 & 0.76 & 0.996 & 0.046 \\
\hline \multicolumn{13}{|l|}{ PASE tertile } \\
\hline Inactive & ref. & & & & ref. & & & & ref. & & & \\
\hline Moderate & 0.79 & 0.50 & 1.24 & 0.30 & 0.87 & 0.54 & 1.36 & 0.53 & 0.82 & 0.50 & 1.35 & 0.44 \\
\hline Active & 0.49 & 0.30 & 0.82 & 0.01 & 0.55 & 0.33 & 0.93 & 0.03 & 0.54 & 0.30 & 0.97 & 0.04 \\
\hline $\begin{array}{l}\text { METS }=\mathrm{N} \\
{ }^{1} \text { Model 1: } \\
{ }^{2} \text { Model 2: } \\
{ }^{3} \text { Every 10 }\end{array}$ & $\begin{array}{l}\text { d for } \\
\text { juste } \\
\text { ange }\end{array}$ & $\begin{array}{l}\text { alent o } \\
\text { e, race } \\
\text { or dial }\end{array}$ & $\begin{array}{l}\text { sk. } \\
\text { ad ge } \\
\text { s, dia }\end{array}$ & $\begin{array}{l}\text { er. } \\
\text { sis vint }\end{array}$ & okin & tor & I, an & ayed & unct & & & \\
\hline
\end{tabular}

questionnaire were more likely to be women (42.7 vs. 34.1 , $\mathrm{p}=0.05)$ and less likely to have a college education (33.8 vs. $44, \mathrm{p}=0.02)$. There was no change in activity category in most recipients (64.8\%). However, 19.7\% had increased their activity by at least one category, and $15.5 \%$ had decreased their activity by at least one category.

We evaluated change in score using several measures. While $42.4 \%$ of the recipients had an increase in score of 20 , only $16.2 \%$ increased it by 70 which is 1 SD of the PASE score. The mean PASE score difference for the cohort was 6.2 (range: -247 to 222.7). We did not find any association between change in PASE score (percentage change or absolute difference) and mortality adjusted for the variables in our full mortality model.

\section{Discussion}

This study reveals that KTRs engage in modest levels of PA at the time of transplantation. Higher PA at the time of kidney transplantation was significantly linked to decreased mortality and death with graft function independent of other established predictors of mortality. During the first year after transplantation, there were modest im- provements in PA. Our study therefore extends the association of PA with all-cause mortality to incident KTRs in an ethnically diverse cohort. We did not find an association between change of PA after transplantation and mortality.

Despite having a wide age range, the recipients in our cohort had PASE scores that were similar to those previously described in the elderly. The PASE scores of the recipients by gender correspond to the PASE scores of 70- to 75-year-old men and 65- to 69-year-old women in the general population. The PASE scores obtained were slightly higher than previously described for a cohort of 39 prevalent dialysis patients $(105.3 \pm 70.2$ vs. $90.3 \pm$ 76.8) likely reflecting their transplant eligibility [6].

Confirming prior studies, age, diabetes, and ESKD vintage were significantly associated with mortality [1113]. The strong relationship between PA and mortality in our study confirms recent studies of physical function among KTRs. Pretransplantation physical functioning, as revealed by the physical function subscale of the Medical Outcomes Study Short-Form 36 questionnaire, was found to be a significant predictor of hospitalization and death among 366 incident dialysis patients who received a transplant within 24 months of starting dialysis [14]. 
In a retrospective study of 402 recipients, Yango et al. [15] found that pretransplant inactivity predicted recipient survival. Recently, Zelle et al. [8] using a different PA questionnaire found a strong association between low PA and all-cause mortality in prevalent KTRs who were transplanted at least a year earlier.

Our findings have important clinical implications. Sedentary lifestyle is associated with increased risk of CVD mortality among individuals with hypertension and diabetes [16-19]. PA is a modifiable risk factor for death. Exercise programs have been shown to improve survival as well as improve glycemic and blood pressure control $[20,21]$. In the kidney transplant population, exercise interventions have been shown to increase peak oxygen intake and muscle strength [22]. The benefits of exercise in kidney replacement therapy participants were notable in physiological parameters, such as $\mathrm{VO}_{2}$ maximum which increased during a 6-month aerobic exercise program and peak exercise heart rate which decreased from a mean $158.6 \pm 21$ to $150.5 \pm 22.7$ in KTRs [23].

Collectively, these findings suggest that the level of PA should be evaluated among kidney transplant candidates and recipients. Counseling kidney transplant candidates and recipients to increase the level of PA could have important health benefits and potentially increase their survival.

Inferences about the impact of changes in PA on the risk of mortality should be made with caution. While it is possible that we were unable to contact the more active participants for follow-up, there was no difference in baseline PA between those that completed a second questionnaire and those without it. One small study of 32 adults noted a $30 \%$ improvement from baseline at 1 year, but no further improvement up to 5 years after transplantation [3].
While our study involved a prospectively followed, large, racially diverse cohort of incident KTRs with long follow-up, this study has limitations including selection bias. KTRs who agreed to participate in the study may not have had equivalent PA compared to individuals who declined participation. Residual confounding is also likely due to inadequate adjustment for unmeasured covariates.

In conclusion, we demonstrated a strong association between low pretransplant PA and increased risk of mortality after transplantation. Clinical exercise intervention trials are needed to determine if training programs preor posttransplant improve mortality outcomes. Evaluation of PA level may be beneficial during the evaluation of candidates for kidney transplantation, and patients listed for transplantation should be encouraged to exercise.

\section{Acknowledgements}

We would like to thank Christine Bonney and Rebecca Pinnelas for their contributions to this paper. The cohort was recruited by funding support in part from NIH K08 DK02626 (S.E.R.). Dr. Rosas's lab is supported by R01 DK080033 and R21 HL086971. The project described was supported by grant No. UL1RR024134 from the National Center for Research Resources. Dr. Reese's effort was supported by K23-DK-078688. The content is solely the responsibility of the authors and does not necessarily represent the official views of the National Center for Research Resources or the National Institutes of Health.

\section{Disclosure Statement}

None of the authors have any conflicts of interest to declare.

\section{References}

1 Kasiske B, Guijarro C, Massy ZA, Wiederkehr JZ: Cardiovascular disease after renal transplantation. J Am Soc Nephrol 1996; 7:158-165.

$\checkmark 2$ Roe P, Wolfe M, Joffe M, Rosas SE: Inflammation, coronary artery calcification and cardiovascular events in incident renal transplant recipients. Atherosclerosis 2010; 212:589-594.

-3 Nielens H, Lejeune TM, Lalaoui A, Squifflet JP, Pirson Y, Goffin E: Increase of physical activity level after successful renal transplantation: a 5 year follow-up study. Nephrol Dial Transplant 2001;16:134-140.
4 Washburn RA, Smith KW, Jette AM, Janney CA: The Physical Activity Scale for the Elderly (PASE): development and evaluation. J Clin Epidemiol 1993;46:153-162.

5 Oken E, Ning Y, Rifas-Shiman SL, Radesky JS, Rich-Edwards JW, Gillman MW: Associations of physical activity and inactivity before and during pregnancy with glucose tolerance. Obstet Gynecol 2006;108:12001207.

6 Johansen KL, Painter P, Kent-Braun JA, Ng AV, Carey S, Da Silva M, Chertow GM: Validation of questionnaires to estimate physical activity and functioning in end-stage renal disease. Kidney Int 2001;59:1121-1127.
7 Ainsworth BE HW, Herrmann SD, Meckes N, Bassett DR Jr, Tudor-Locke C, Greer JL, Vezina J, Whitt-Glover MC, Leon AS: The Compendium of Physical Activities Tracking Guide. Healthy Lifestyles Research Center, College of Nursing and Health Innovation, Arizona State University, 2011. https://sites. google.com/site/compendiumofphysicalactivities/ (accessed May 2011)

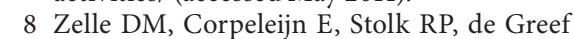
MH, Gans RO, van der Heide JJ, Navis G, Bakker SJ: Low physical activity and risk of cardiovascular and all-cause mortality in renal transplant recipients. Clin J Am Soc Nephrol 2011;6:898-905. 
-9 Harada ND, Chiu V, King AC, Stewart AL: An evaluation of three self-report physical activity instruments for older adults. Med Sci Sports Exerc 2001;33:962-970.

-10 Janney CA, Cauley JA, Cawthon PM, Kriska AM: Longitudinal physical activity changes in older men in the osteoporotic fractures in men study. J Am Geriatr Soc 2010;58:11281133.

- 11 Cosio FG, Alamir A, Yim S, Pesavento TE, Falkenhain ME, Henry ML, Elkhammas EA, Davies EA, Bumgardner GL, Ferguson RM: Patient survival after renal transplantation: I. The impact of dialysis pre-transplant. Kidney Int 1998;53:767-772.

-12 Witczak BJ, Jenssen T, Endresen K, Roislien J, Hartmann A: Risk factors for mortality in diabetic nephropathy patients accepted for transplantation. Transplantation 2007;84: 356-361.

-13 Kim SJ, Schaubel DE, Fenton SS, Leichtman AB, Port FK: Mortality after kidney transplantation: a comparison between the United States and Canada. Am J Transplant 2006; 6:109-114.
4 Kutner NG, Zhang R, Bowles T, Painter P: Pretransplant physical functioning and kidney patients' risk for posttransplantation hospitalization/death: evidence from a national cohort. Clin J Am Soc Nephrol 2006; 1:837-843.

15 Yango AF, Gohh RY, Monaco AP, Reinert SE, Gautam A, Dworkin LD, Morrissey PE: Excess risk of renal allograft loss and early mortality among elderly recipients is associated with poor exercise capacity. Clin Nephrol 2006;65:401-407.

16 Engstrom G, Hedblad B, Janzon L: Hypertensive men who exercise regularly have lower rate of cardiovascular mortality. J Hypertens 1999; 17:737-742.

17 Tanasescu M, Leitzmann MF, Rimm EB, Hu FB: Physical activity in relation to cardiovascular disease and total mortality among men with type 2 diabetes. Circulation 2003;107: 2435-2439.

$18 \mathrm{Hu}$ FB, Stampfer MJ, Solomon C, Liu S, Colditz GA, Speizer FE, Willett WC, Manson JE: Physical activity and risk for cardiovascular events in diabetic women. Ann Intern Med 2001;134:96-105.
19 Physical activity and cardiovascular health. NIH consensus development panel on physical activity and cardiovascular health. JAMA 1996;276:241-246.

20 Fossum E, Gleim GW, Kjeldsen SE, Kizer JR, Julius S, Devereux RB, Brady WE, Hille DA, Lyle PA, Dahlof B: The effect of baseline physical activity on cardiovascular outcomes and new-onset diabetes in patients treated for hypertension and left ventricular hypertrophy: the LIFE study. J Intern Med 2007;262:439-448.

21 Knowler WC, Barrett-Connor E, Fowler SE, Hamman RF, Lachin JM, Walker EA, Nathan DM: Reduction in the incidence of type 2 diabetes with lifestyle intervention or metformin. N Engl J Med 2002;346:393-403.

22 Painter PL, Hector L, Ray K, Lynes L, Dibble S, Paul SM, Tomlanovich SL, Ascher NL: A randomized trial of exercise training after renal transplantation. Transplantation 2002; 74:42-48.

23 Violan MA, Pomes T, Maldonado S, Roura G, De la Fuente I, Verdaguer T, Lloret R, Torregrosa JV, Campistol JM: Exercise capacity in hemodialysis and renal transplant patients. Transplant Proc 2002;34:417-418. 